\title{
DES MAINS DÉLICATES POUR DES FRAISES AMÈRES
}

Emmanuelle Hellio

GISTI | « Plein droit »

$2008 / 3 n^{\circ} 78 \mid$ pages 34 à 38

ISSN 0987-3260

Article disponible en ligne à l'adresse :

https://www.cairn.info/revue-plein-droit-2008-3-page-34.htm

\section{Pour citer cet article :}

Emmanuelle Hellio, "Des mains délicates pour des fraises amères 》, Plein droit 2008/3 (n 78), p. 34-38. DOI 10.3917/pld.078.0034

Distribution électronique Cairn.info pour GISTI.

(c) GISTI. Tous droits réservés pour tous pays.

La reproduction ou représentation de cet article, notamment par photocopie, n'est autorisée que dans les limites des conditions générales d'utilisation du site ou, le cas échéant, des conditions générales de la licence souscrite par votre établissement. Toute autre reproduction ou représentation, en tout ou partie, sous quelque forme et de quelque manière que ce soit, est interdite sauf accord préalable et écrit de l'éditeur, en dehors des cas prévus par la législation en vigueur en France. Il est précisé que son stockage dans une base de données est également interdit. 


\begin{abstract}
Dans le marché international agricole, l'Andalousie est la première zone exportatrice de produits maraîchers pour l'ensemble de l'Europe. L'immigration est considérée comme un des facteurs clé de la rentabilité du système. Intégrés dans une agriculture industrielle mondialisée, les agriculteurs recherchent, depuis le début des années 2000, l'intrant supplémentaire nécessaire à leur production, une main-d'œuvre étrangère la plus flexible possible. Les exploitants importent des travailleurs pour exporter des fraises.
\end{abstract}

\title{
Des mains délicates pour des fraises amères
}

\section{Emmanuelle Hellio}

Stagiaire SOC-Confédération paysanne dans le cadre du programme européen agriculture paysanne et travailleurs migrants saisonniers.

À l'ouest de la communauté autonome d'Andalousie, la province de Huelva s'est spécialisée depuis les années 80 dans la culture de la fraise. Aujourd'hui, plus de 7000 hectares de serre sont cultivés sur d'anciennes pinèdes publiques. Le cycle de production commence à la fin de l'été par la stérilisation des sols au bromure de méthyle. En octobre, les fraisiers qui ont grandi dans le froid des pépinières de Castilla y León, sont transplantés à Huelva. Ils produisent dès la fin du mois de décembre et jusqu'en juin des fraises qui seront exportées dans toute l'Europe. Il s'agit d'une culture hydroponique, le sol des serres n'apporte aucun élément nutritif aux plants. Cette production intensive dépend donc des multinationales qui fournissent les plastiques, les engrais et les pesticides indispensables à ce mode de culture. Même les plants viennent d'ailleurs. La plupart des agriculteurs cultivent la fraise Camarosa, une variété créée par une université californienne et dont la plantation suppose de payer des royalties à hauteur de 1800 euros par hectare et par an.

Pour rester rentable malgré l'importance des coûts intermédiaires, la production de «l'or rouge $\gg$ ne peut cependant se maintenir que si les agriculteurs disposent d'une main-d'œuvre nombreuse, bon marché et disponible tout au long de la récolte. En
2000, les organisations patronales font le constat d'un manque de travailleurs. Les journaliers andalous qui récoltaient les fraises depuis les années 80 se détournent toujours davantage d'un des secteurs les plus précaires et les moins payés d'Espagne. Insensiblement, durant les années 90, ce sont des hommes originaires d'Afrique noire ou du Maghreb, issus d'une immigration spontanée qui les ont remplacés dans les champs. Malgré la présence de ces hommes et peut être dans le but d'éviter les risques d'emploi massif d'une main-d'œuvre majoritairement illégale, les organisations patronales décident de faire venir temporairement de l'étranger les saisonniers dont ils ont besoin. Ils utilisent à cette fin une clause de la ley de extranjería, la loi espagnole sur l'entrée et le séjour des étrangers. Votée en 


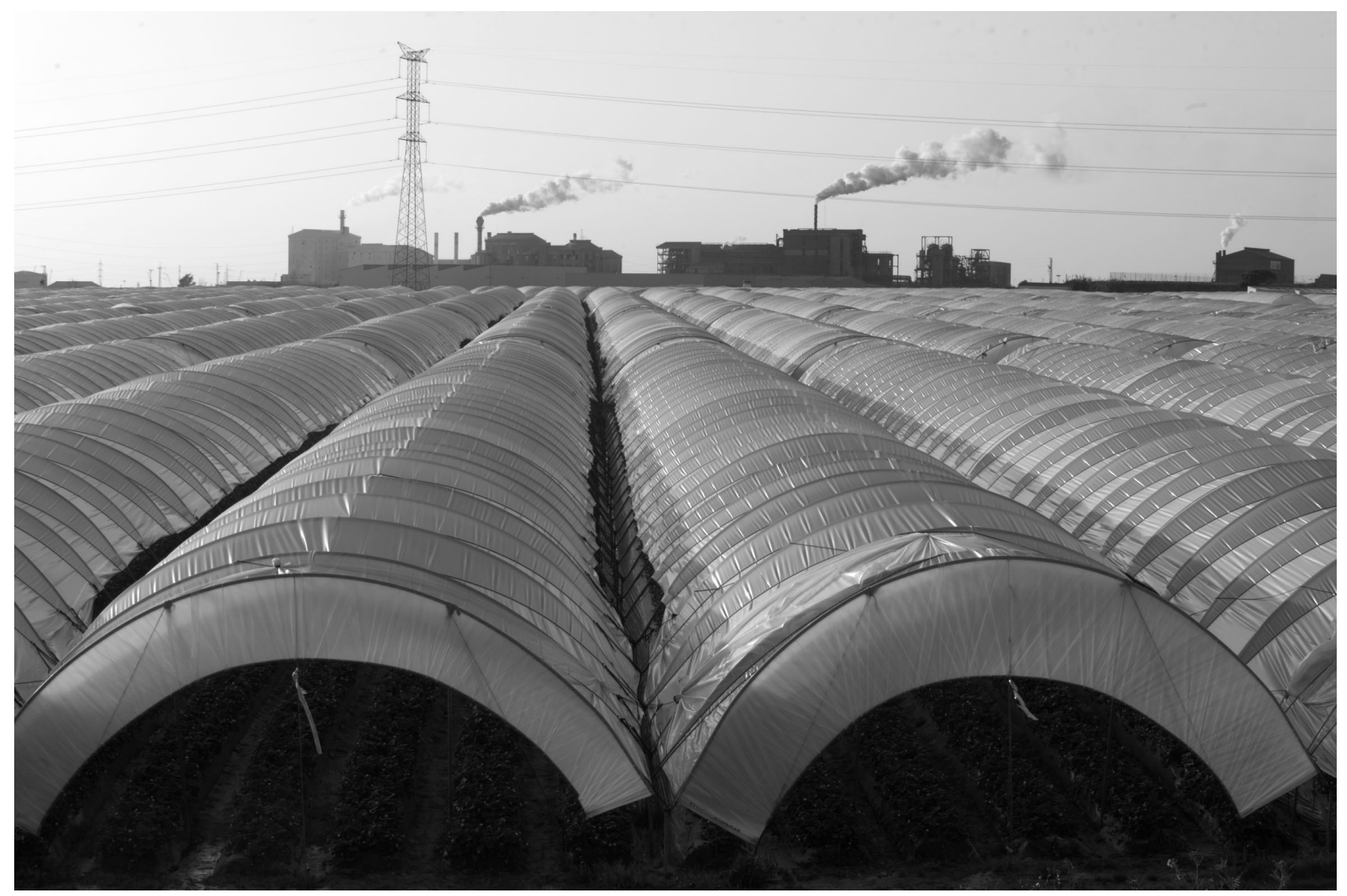

Photo E. Hellio

$2000^{(1)}$, elle offre la possibilité aux employeurs qui ne trouvent pas localement les travailleurs dont ils ont besoin, de recruter des ressortissants de pays non communautaires, à la condition que ces étrangers se trouvent dans leur pays au moment de la signature du contrat. Acheminés jusqu'en Espagne pour le temps de la récolte, ils devront ensuite rentrer dans leur pays à la fin du contrat saisonnier. C'est la naissance de la contratación en origen qu'on traduira par « recrutement en origine ». Intégrés dans une agriculture industrielle mondialisée, les agriculteurs vont chercher l'intrant supplémentaire nécessaire à leur production : une main-d'œuvre étrangère la plus flexible possible. Dans ce système agricole où l'immigration est considérée comme un des facteurs clé de la rentabilité, les exploitants impor- tent des travailleurs pour exporter des fraises.

En 2000, quelques organisations patronales mènent une première expérience pilote avec des travailleuses polonaises. Devant le succès de l'opération, toutes les coopératives, organisations et syndicats se tournent vers ce mode d'embauche et les chiffres des « recrutés en origine » vont augmenter chaque année de façon exponentielle : 1200 en 2001, 12000 en 2003, 24000 en 2004 pour atteindre 35000 en 2008. Selon la ley de extranjería modifiée en 2003 , le recrutement doit être mené de préférence dans des pays ayant signé des accords de régulation des flux migratoires avec l'Espagne. À part cette contrainte légale, ce sont les organisations patronales qui choisissent les pays de recrutement. Elles se tournent vers « des pays ruraux, au PIB/ habitant faible » et qui connaissent un taux de chômage élevé afin d'assurer un différentiel de salaire important. Pendant ces huit années, les exploitants ont sélectionné des travailleurs en Pologne, en Roumanie, en Bulgarie et au Maroc. Cette année, un recrutement a également eu lieu en Ukraine bien que ce pays n'ait signé aucun accord de régulation des flux migratoires, et une expérience pilote a été menée au Sénégal. Chaque année, les organisations patronales estiment les besoins de main-d'œuvre du secteur et chargent l'agence pour l'emploi des pays d'origine de mener une présélection. Elles se rendent ensuite elles-mêmes dans les pays pour finaliser le recrutement. À la recherche de ce qu'ils appellent le «profil adéquat à la fraise », les employeurs sélectionnent : - des femmes, parce qu'elles 
« génèrent moins de conflits, elles ont les mains plus délicates, elles sont plus travailleuses, et plus bumbles »; - avec une expérience dans l'agriculture; - et ayant dans le pays des charges familiales et des enfants en bas âge, afin de s'assurer de leur motivation au travail et de leur retour au pays d'origine une fois la saison terminée.

Bien que le recrutement se déroule dans tous les pays cités plus haut, les exploitants peuvent préciser aux organisations patronales la nationalité du travailleur désiré. On peut constater dans leurs discours une préférence pour les femmes des pays de l'Est par rapport aux africaines «qui n'ont pas la même culture, la même religion et ne savent pas parler espagnol [...]. Le problème des travailleuses marocaines, c'est que quand je dois les répartir aux agriculteurs, ils n'en veulent pas. Des 350 de cette année, il m'en reste 50 sur les bras... et je dois encore placer toutes les Sénégalaises. »(2) Selon le représentant d'une des plus grandes coopératives du secteur, ces préférences nationales, qui virent parfois au préjugé ethnique («Le

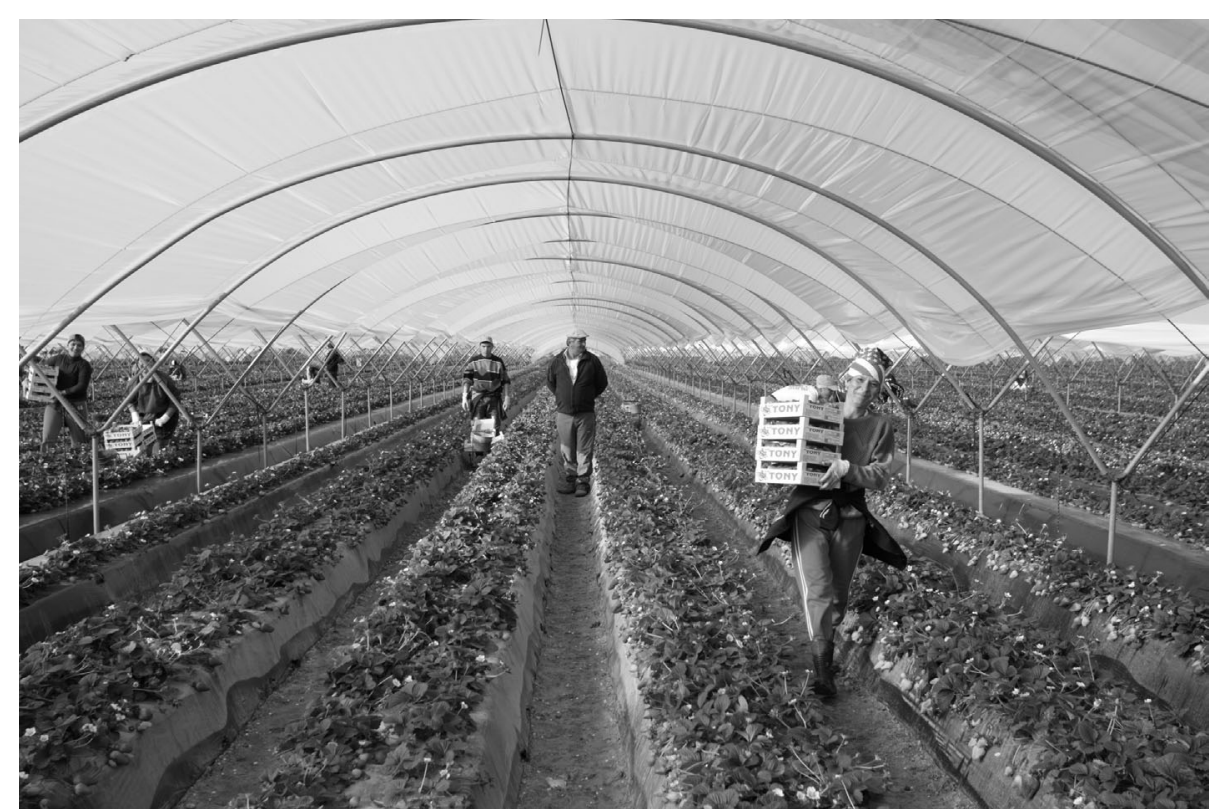

Photo E. Hellio travailleur marocain est plus polémique parce qu'il a d'autres coutumes, une autre culture, différente de la nôtre. Il lui coûte plus de cohabiter avec des races différentes de la sienne $[\ldots] \gg)$, sont « légitimes » dans la mesure où, «dans une économie de marché, l'administration et les entreprises essayent d'amener les personnes les moins polémiques possible. $\gg^{(3)}$

Ces femmes participent à toutes les tâches de la campagne de fraises : récolte, emballage dans les entrepôts de conditionnement, arrachage des plastiques, épandage des pesticides, etc. Dans la mesure où ce sont les propres employeurs qui estiment les besoins en travailleurs du secteur et que, selon la convention collective, les travailleurs ne sont payés que les jours où ils travaillent, ils peuvent se permettre d'importer une main-d'œuvre excédentaire qui sera disponible pour les pics de production mais travaillera peu de jours au début et à la fin de la saison.

Sur certains contrats, on peut lire que «du fait du caractère discontinu de l'activité, le travail à réaliser dépend de l'état des récoltes, du degré de maturation, de la continuité des commandes et des intempéries (pluie, grêle, gelée, etc). Le travail ne sera pas continu même pendant la durée du contrat. Au contraire, la quantité de journées [travaillées] variera mensuellement en proportion du produit apte à être semé, traité ou récolté. Le travail effectif dépendra des circonstances citées. 》

Prenant acte du sous emploi de ces travailleuses (en moyenne quatorze jours par mois sur toute la saison), le gouvernement a introduit une «garantie d'activité continue » dans le dernier accord qui régule le contingent 2008. «Les offres d'emploi de caractère temporaire garantiront au travailleur recruté une activité continue pendant toute la durée de l'autorisation sollicitée » (dix-huit jours minimum par mois). Dans la pratique, ce seuil n'est pas contrôlé par les administrations et est donc peu respecté.

En réalité, le « recrutement en origine » porte en lui la docilité du travailleur. Lorsqu'il obtient son contrat, l'étranger commence par signer un engagement de retour. Il se voit alors délivrer par la direction générale de l'immigration «une autorisation de résidence temporaire et de travail où seront indiqués le secteur géographique et le secteur d'activité autorisés ainsi que la durée de cette autorisation qui coïncidera avec la durée du contrat ${ }^{(4)}$, de trois à neuf mois. Cette dépendance du séjour de l'étranger au contrat de travail protège le système contre toute velléité de revendication ou d'organisation collective. Lorsqu'elle perd son emploi, une personne venue avec un «contrat en origine » perd aussi son droit au séjour. Ensuite, soit elle rentre dans son pays, soit elle devient une sans-papiers. De ce fait, les 


\title{
«Des équipes interculturelles »
}

\author{
« Je fais des équipes de travail interculturelles, à cha-
} que rang je mets une nationalité différente : là un Marocain, ensuite un Roumain, un du Mali, une Polonaise, une autre Roumaine. Je les mélange. Je me suis rendu compte que c'est beaucoup mieux pour le rythme de travail. Ceux avec qui j'ai eu le plus de problèmes c'était les Roumains. Quand l'un d'entre eux ramassait rapidement, les autres l'arrêtaient: "calme toi, tout ce que tu fais c'est faire gagner de l'argent au chef". Quand ils sont tous de la même nationalité, ils parlent la même langue. Tu ne peux pas les comprendre et ils se sentent forts. Mais quand tu les mélanges, ça va mieux. D'abord, ils ne se comprennent pas et ensuite ils ne se disputent pas, ou alors s'ils se disputent, c'est tout bénéf' pour moi, parce si le Marocain se fâche avec le Roumain, il essaiera de ramasser plus que l'autre. Avant j'avais beaucoup de travailleurs roumains, maintenant j'essaye d'en avoir de tous les pays. »

Entretien réalisé avec un exploitant de 4 hectares de serre à Palos de la frontera.

« recrutées en origine » sont totalement liées à leur employeur. Dans la majorité des cas, on ne leur paye pas leurs heures supplémentaires, ni les jours fériés. La dépendance est accentuée par le fait que la plupart de ces saisonnières sont logées au sein des exploitations souvent isolées des villages de plusieurs kilomètres alors même qu'elles ne disposent d'aucun moyen de transport. Dans les baraquements, on les regroupe par nationalité. Il arrive que certaines entreprises installent leur entrepôt de conditionnement au cœur même des serres.

Le système des « contrats en origine » est le prototype même de l'utilitarisme migratoire en ce sens qu'il permet de disposer légalement de travailleurs aussi précaires que pourraient l'être des «sans-papiers ». Cependant, l'existence de ce réservoir de main-d'œuvre légal ne signifie pas que les patrons n'emploient plus d'autres travailleurs étrangers. Il faut prendre garde à ne pas établir une frontière rigide entre les travailleurs légaux que sont les « recrutés en origine », et les « sans-papiers ». En réalité, un étranger ayant signé un « contrat en origine » est tout au long de son séjour en «sursis de clandestinité ». S'il décide de rester ou de travailler dans un autre secteur, il sera alors «illégalisé ». Selon les évaluations des employeurs, plus de 100000 travailleurs agricoles participeraient chaque année à la campagne de fraises. En plus des « recrutés en origine », cette main-d'œuvre est composée d'autres étrangers aux statuts administratifs divers : « sanspapiers », ressortissants communautaires de pays entrés récemment dans l'Union européenne comme les Polonais venus initialement avec des «contrats en origine » mais qui peuvent désormais circuler et travailler librement en Espagne, ressortissants communautaires de pays soumis à la période transitoire, principalement des Roumains et des Bulgares $^{(5)}$.

Les employeurs disposent donc d'une main-d'œuvre nombreuse et diversifiée qui leur permet de faire jouer une concurrence statutaire afin d'intensifier les rythmes de travail ou de baisser les salaires. Ainsi des étrangers sans autorisation de séjour ou de travail seront disposés à accepter un jornal(6) inférieur aux trente-cinq euros de la convention collective. Certains employeurs mettent même en œuvre une concurrence entre les différentes nationalités dont ils disposent dans leurs équipes de travail pour intensifier la productivité.

$\mathrm{Si}$, sur le terrain, on peut observer la coexistence des « contrats en origine » avec d'autres formes d'embauche de travailleurs étrangers, les institutions et les organisations patronales mettent toujours en avant le modèle du « recrutement en origine », présenté comme une migration légale et temporaire et, de ce fait, acceptable. Cependant, à trop se persuader du provisoire de ces déplacements, en invoquant constamment l'effectivité du retour des travailleurs dans leur pays d'origine, les promoteurs de la migration circulaire en arrivent à oublier que beaucoup de femmes restent en Espagne. Et lorsque certains en font le constat, ce n'est que pour déplorer les « $50 \%$ de pertes de Marocaines ${ }^{(7)}$, les chiffres de « fugue » des Sénégalaises qui «se sont échappées », ou alors pour trouver les moyens de réduire ces «risques d'évasion ». Ce champ lexical de l'emprisonnement indique qu'aujourd'hui, l'enfer- 
mement des étrangers ne se limite pas à l'exclusion géographique du territoire européen, ou à la rétention dans des centres. Il comprend aussi l'enfermement dans des statuts administratifs vecteurs de servitude, qui permettent à des travailleurs de se trouver et de travailler en Europe sans pour autant avoir accès aux droits.

Le 17 et 18 novembre 2007, au Portugal, se tenait la première réunion ministérielle euro-méditerranéenne sur les migrations. L'objectif de cette rencontre était de définir les futurs axes d'une politique migratoire dans l'espace euro-méditerranéen. Parmi les conclusions de la réunion, on note la volonté de «promouvoir les opportunités de migration légale dans la région, en prenant en compte les besoins des marchés $d u$ travail respectifs ». Les ministres se proposent d' «analyser les possibilités de faciliter et de simplifier les procédures de migration légale pour les travailleurs demandeurs. Ces efforts se concentreraient sur les différentes catégories de travailleurs légaux et pourraient également inclure différentes formes de mobilité comme les migrations circulaires et temporaires. 》

Face à cette tendance européenne ${ }^{\left({ }^{8}\right)}$, il serait bon d'interroger la catégorie juridique des «saisonniers » et de dénoncer la logique migratoire actuelle de l'immigration choisie qui réduit les étrangers venant chez nous à leur compétence ou à leur force physique de travail sans qu'ils aient droit à l'installation. Une politique qui condamne des personnes à une existence partielle, réduite au temps et à la quantité de travail fournis, où les droits de l'homme n'existent pas.

(1) Article 42, Ley orgánica 4/2000.

(2) Entretien réalisé avec E D.C, responsable du recrutement en origine à la Coag (syndicat agricole), le 15/11/2007 à Palos de la frontera.

(3) Article du quotidien El País 08/03/02, « La patronal de la fresa rechaza a los trabajadores marroquíes porque son "polémicos" 》 (L'organisation patronale de la fraise rejette les travailleurs marocains parce qu'ils sont "polémiques".)

(4) Accord de régulation du contingent 2008. (5) Les Roumains et les Bulgares jouissent depuis 2007 de la liberté de circulation mais devront attendre le ${ }^{\text {er }}$ janvier 2009 pour être dispensés d'autorisation de travail.

(6) Salaire d'une journée de travail. Les travailleurs agricoles qui héritent des modes d'emploi des anciens journaliers ne sont pas payés à l'heure mais à la journée de travail.

(7) Entretien réalisé avec E D.C, responsable du recrutement en origine à la Coag (syndicat agricole), le 15/11/2007 à Palos de la frontera.

(8) 18, 19 Novembre 2007). Agreed Ministerial Conclusions. First Euro-Mediterranean Ministerial Meeting on Migration, Algarve, Portugal.

\section{«Un jour, on a fait grève, on n'a travaillé que 7 heures. Après, il n'a pas renouvelé le contrat »}

«Dans les serres, on travaille tous les jours du lundi au dimanche. Quand la campagne devient forte, il n'y a plus de jours de repos, et cela dure deux ou trois mois. Si tu t'arrêtes une journée, ils te punissent en t'interdisant de travailler les jours suivants. Les coopératives, c'est la même chose (...). Pendant le pic de production, on y travaille jusqu'à 13 heures tous les jours. (...)

" Je suis venue pour la première fois en 2004. J'ai fait deux saisons, mais en 2006, je n'ai pas pu venir parce que le chef ne m'a pas laissé "entrer". Il a retiré dix personnes des champs, il ne m'a pas renouvelé le contrat, à moi et à neuf autres filles. Je vais te raconter pourquoi. [Tous les jours], nous faisions des heures supplémentaires, une heure, une heure et demie, deux heures. On était fatiguées, personne ne peut faire autant d'heures le dos courbé sans se relever, mais il ne voulait rien savoir. Alors un jour on a fait grève, mais une grève spéciale : on a travaillé sept heures, et ensuite on est sorties des serres. C'est pour cela qu'il ne nous a pas voulues l'année suivante. (...)

«Ici, tu es là pour travailler, travailler et travailler, c'est tout. Tu n'as rien à dire, tu ne peux pas avoir de copain ou sortir, quand nous le faisions c'était en secret. Le chef contrôlait ta vie, ta façon de travailler, où tu allais, comment tu t'habillais. Ici les chefs disent «si tu ne couches pas avec moi, tu t'en vas. Cette nuit, je dors avec toi. » 